\title{
Rate-Memory Trade-off for the Two-User Broadcast Caching Network with Correlated Sources
}

\author{
Parisa Hassanzadeh, Antonia M. Tulino, Jaime Llorca, Elza Erkip
}

\begin{abstract}
This paper studies the fundamental limits of caching in a network with two receivers and two files generated by a two-component discrete memoryless source with arbitrary joint distribution. Each receiver is equipped with a cache of equal capacity, and the requested files are delivered over a shared errorfree broadcast link. First, a lower bound on the optimal peak rate-memory trade-off is provided. Then, in order to leverage the correlation among the library files to alleviate the load over the shared link, a two-step correlation-aware cache-aided coded multicast (CACM) scheme is proposed. The first step uses GrayWyner source coding to represent the library via one common and two private descriptions, such that a second correlationunaware multiple-request CACM step can exploit the additional coded multicast opportunities that arise. It is shown that the rate achieved by the proposed two-step scheme matches the lower bound for a significant memory regime and it is within half of the conditional entropy for all other memory values.
\end{abstract}

\section{INTRODUCTION AND SETUP}

The use of caching at the wireless network edge has emerged as a promising approach to efficiently increase the capacity of wireless access networks. There have been extensive studies characterizing the fundamental rate-memory tradeoff in a broadcast caching network with a library composed of independent content [1]-[3]. More recently, [4]-[6] have studied the rate-memory trade-off when delivering correlated files. In [4], the authors consider a single-receiver multiplefile network with lossy reconstructions and characterize the trade-offs between rate, cache capacity, and reconstruction distortions. They extend the analysis to a scenario with two receivers and one cache, in which local caching gains can be explored. The works in [5] and [6] consider a setting with an arbitrary number of files and receivers each having a cache. A practical correlation-aware scheme is proposed in [5], in which content is cached according to both the popularity of files and their correlation with the rest of the library. Then, the requested content is delivered via a multicast codeword composed of compressed versions of the requested files. Alternatively, the work in [6] addresses the dependency among content files by first compressing the correlated library, which is then treated as a library of independent files by a conventional cache-aided coded multicast scheme.

This work has been supported by NSF grant \#1619129.

P. Hassanzadeh and E. Erkip are with the ECE Department of New York University, Brooklyn, NY. Email: \{ph990, elza $\}$ nyu.edu

J. Llorca and A. Tulino are with Bell Labs, Nokia, Holmdel, NJ, USA. Email: \{jaime.llorca, a.tulino\}@ nokia-bell-labs.com

A. Tulino is with the DIETI, University of Naples Federico II, Italy. Email: \{antoniamaria.tulino\}@unina.it
In this paper, by focusing on a two-user two-file setting, we are able to characterize the optimal peak rate-memory tradeoff of the broadcast caching network with correlated sources. To this end, we first provide a lower bound on the optimal peak rate-memory trade-off, which is derived using a cutset argument on the corresponding cache-demand augmented graph [7]. The lower bound improves the best known bound for correlated sources given in [8], and when particularized to independent sources, matches the corresponding best known bound derived in [9]. We then propose a two-step scheme, in which the source files are first encoded based on the GrayWyner network [10], and in the second step, they are cached and delivered through a multiple-request correlation-unaware cache-aided coded multicast scheme. In the rest of the paper, we discuss the optimality of the proposed two-step scheme by characterizing a lower bound on the rate-memory tradeoff for this class of schemes, and comparing it with the lower bound on the optimal trade-off. We identify the set of operating points in the Gray-Wyner region [10], [11], for which a twostep scheme is optimal over a range of cache capacities, and approximates the optimal rate to within half of the conditional entropy for all cache sizes.

The paper is organized as follows. Sec. II presents the information-theoretic problem formulation. In Sec. III we introduce a class of two-step schemes based on the GrayWyner network. The lower bounds for the optimal and twostep schemes are provided in Sec. IV, and later used to establish the optimality of an achievable two-step scheme proposed in Sec. V A After analyzing an illustrative example in Sec. V-A, the paper is concluded in Sec. VI

\section{Network Model And Problem Formulation}

We consider a broadcast caching network composed of one sender with access to a library of two files generated by a 2-component discrete memoryless source (2-DMS). The 2-DMS model $\left(\mathcal{X}_{1} \times \mathcal{X}_{2}, p\left(x_{1}, x_{2}\right)\right)$ consists of two finite alphabets $\mathcal{X}_{1}, \mathcal{X}_{2}$ and a joint pmf $p\left(x_{1}, x_{2}\right)$ over $\mathcal{X}_{1} \times \mathcal{X}_{2}$. The 2-DMS generates and i.i.d. random process $\left\{\mathrm{X}_{1 i}, \mathrm{X}_{2 i}\right\}$ with $\left(\mathrm{X}_{1}, \mathrm{X}_{2}\right) \sim p\left(x_{1}, x_{2}\right)$. For a block length $n$, the two library files are represented by sequences $\mathrm{X}_{1}^{n}=\left(\mathrm{X}_{11}, \ldots, \mathrm{X}_{1 n}\right)$ and $\mathrm{X}_{2}^{n}=\left(\mathrm{X}_{21}, \ldots, \mathrm{X}_{2 n}\right)$, respectively, where $\mathrm{X}_{1}^{n} \in \mathcal{X}_{1}^{n}$ and $\mathrm{X}_{2}^{n} \in \mathcal{X}_{2}^{n}$. The sender communicates with two receivers $r_{1}$ and $r_{2}$ over a shared error-free broadcast link. Each receiver is equipped with a cache of size $n M$ bits, where $M$ denotes the (normalized) cache capacity.

We assume that the system operates in two phases: a caching phase and a delivery phase. During the caching phase, which 
takes place at off-peak hours when network resources are abundant, the receiver caches are filled with functions of the library files, such that during the delivery phase, when receiver demands are revealed and resources are limited, the sender broadcasts the shortest possible codeword that allows each receiver to losslessy recover its requested file. We refer to the overall scheme as a cache-aided coded multicast scheme (CACM). Given a realization of the library, $\left\{X_{1}^{n}, X_{2}^{n}\right\}$, a CACM scheme consists of the following components:

- Cache Encoder: During the caching phase, the cache encoder designs the cache content of receiver $r_{i}$ using a mapping $f_{r_{i}}^{\mathfrak{C}}: \mathcal{X}_{1}^{n} \times \mathcal{X}_{2}^{n} \rightarrow\left[1: 2^{n M}\right)$. The cache configuration of receiver $r_{i}$ is denoted by $Z_{r_{i}}=f_{r_{i}}^{\mathfrak{C}}\left(X_{1}^{n}, X_{2}^{n}\right)$.

- Multicast Encoder: During the delivery phase, each receiver requests a file from the library. The demand realization, denoted by $\mathbf{d}=\left(d_{r_{1}}, d_{r_{2}}\right) \in \mathcal{D} \equiv\{1,2\}^{2}$, where $d_{r_{i}} \in\{1,2\}$ denotes the index of the file requested by receiver $r_{i}$, is revealed to the sender, which then uses a fixed-to-variable mapping $f^{\mathfrak{M}}: \mathcal{D} \times\left[1: 2^{n M}\right) \times[1:$ $\left.2^{n M}\right) \times \mathcal{X}_{1}^{n} \times \mathcal{X}_{2}^{n} \rightarrow \mathcal{Y}^{\star}$ to generate and transmit a multicast codeword $Y_{\mathbf{d}}=f^{\mathfrak{M}}\left(\mathbf{d},\left\{Z_{r_{1}}, Z_{r_{2}}\right\},\left\{X_{1}^{n}, X_{2}^{n}\right\}\right)$ over the shared link 11 The codeword $Y_{\mathbf{d}}$ is designed for each demand realization according to the cache content, library files, and joint distribution $p\left(x_{1}, x_{2}\right)$, to enable almost-lossless reconstruction of the requested files.

- Multicast Decoders: Each receiver $r_{i}$ uses a mapping $g_{r_{i}}^{\mathfrak{M}}: \mathcal{D} \times \mathcal{Y}^{\star} \times\left[1: 2^{n M}\right) \rightarrow \mathcal{X}_{d_{r_{i}}}^{n}$ to recover its requested file, $X_{d_{r_{i}}}^{n}$, using the received multicast codeword and its cache content as $\widehat{X}_{d_{r_{i}}}^{n}=g_{r_{i}}^{\mathfrak{M}}\left(\mathbf{d}, Y_{\mathbf{d}}, Z_{d_{r_{i}}}\right)$.

The worst-case probability of error of a CACM scheme is given by

$$
P_{e}^{(n)}=\max _{\mathbf{d}} \max _{r_{i}} \mathbb{P}\left(\widehat{X}_{d_{r_{i}}}^{n} \neq X_{d_{r_{i}}}^{n}\right) .
$$

In this paper, we focus on the peak multicast rate, corresponding to the worst-case demand,

$$
R^{(n)}=\max _{\mathbf{d}} \frac{\mathbb{E}\left[L\left(Y_{\mathbf{d}}\right)\right]}{n},
$$

where $L(Y)$ denotes the length (in bits) of the multicast codeword $Y$, and the expectation is over the library files.

Definition 1: A peak rate-memory pair $(R, M)$ is achievable if there exists a sequence of CACM schemes for cache capacity $M$ and increasing block length $n$, such that $\lim _{n \rightarrow \infty} P_{e}^{(n)}=0$, and $\lim \sup _{n \rightarrow \infty} R^{(n)} \leq R$.

Definition 2: The peak rate-memory region, $\mathfrak{R}^{*}$, is the closure of the set of achievable peak rate-memory pairs $(R, M)$, and the optimal peak rate-memory function is

$$
R^{*}(M)=\inf \left\{R:(R, M) \in \mathfrak{R}^{*}\right\} .
$$

\section{GRAY-WYNER CACM SCHEME}

In this section, we describe a class of schemes based on a two-step lossless source coding setup, as depicted in Fig. 1. The first step involves compressing the library via Gray-Wyner

\footnotetext{
${ }^{1}$ We use $\star$ to indicate variable length.
}

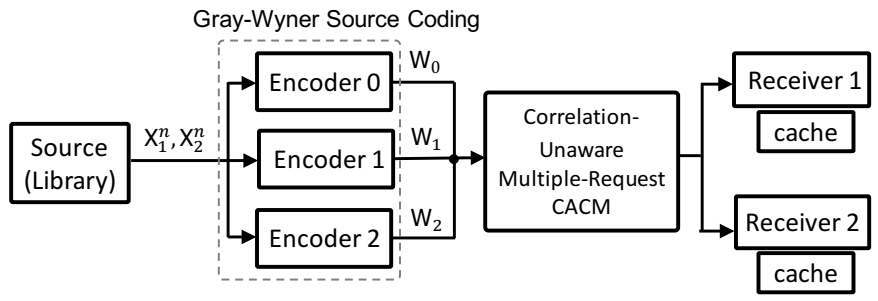

Fig. 1: Gray-Wyner CACM scheme, composed of a first GrayWyner source coding step, and a second correlation-unaware multiple-request CACM step.

source coding, and the second step is a correlation-unaware multiple-request CACM scheme. We refer to this scheme as Gray-Wyner Cache-Aided Coded Multicast (GW-CACM).

Gray-Wyner source coding, depicted in Fig. 1. is a distributed lossless source coding setup in which a 2-DMS $\left(\mathrm{X}_{1}, \mathrm{X}_{2}\right)$ is represented by three descriptions $\left\{\mathrm{W}_{0}, \mathrm{~W}_{1}, \mathrm{~W}_{2}\right\}$, where $\mathrm{W}_{0} \in\left[1: 2^{n R_{0}}\right), \mathrm{W}_{1} \in\left[1: 2^{n R_{1}}\right)$, and $\mathrm{W}_{2} \in[1:$ $\left.2^{n R_{2}}\right)$. File $d_{r_{1}}$ can be losslessly recovered from descriptions $\left(\mathrm{W}_{0}, \mathrm{~W}_{d_{r_{1}}}\right)$, and file $d_{r_{2}}$ can be losslessly recovered from descriptions $\left(\mathrm{W}_{0}, \mathrm{~W}_{d_{r_{2}}}\right)$, both asymptotically, as $n \rightarrow \infty$.

As shown in [11], the Gray-Wyner rate region is the closure of the union over $\mathrm{U}$ of $\mathfrak{S}_{G W}(\mathrm{U})$, where $\mathfrak{S}_{G W}(\mathrm{U})$ denotes the set of rate triplets $\left(R_{0}, R_{1}, R_{2}\right)$ such that

$$
\begin{aligned}
& R_{0} \geq I\left(\mathrm{X}_{1}, \mathrm{X}_{2} ; \mathrm{U}\right), \\
& R_{1} \geq H\left(\mathrm{X}_{1} \mid \mathrm{U}\right), \\
& R_{2} \geq H\left(\mathrm{X}_{2} \mid \mathrm{U}\right),
\end{aligned}
$$

given a conditional pmf $p\left(u \mid x_{1}, x_{2}\right)$ with $|\mathcal{U}| \leq\left|\mathcal{X}_{1}\right| .\left|\mathcal{X}_{2}\right|+2$.

For a given $\mathrm{U}$ and a rate triplet $\left(R_{0}, R_{1}, R_{2}\right) \in \mathfrak{S}_{G W}(\mathrm{U})$, a GW-CACM scheme consists of:

- Gray-Wyner Encoder: Given a library realization $\left\{X_{1}^{n}\right.$, $\left.X_{2}^{n}\right\}$, the Gray-Wyner encoder at the sender computes three descriptions $\left\{\mathrm{W}_{0}, \mathrm{~W}_{1}, \mathrm{~W}_{2}\right\}$ using a mapping $f^{G W}$ : $\mathcal{X}_{1}^{n} \times \mathcal{X}_{2}^{n} \rightarrow\left[1: 2^{n R_{0}}\right) \times\left[1: 2^{n R_{1}}\right) \times\left[1: 2^{n R_{2}}\right)$.

- Correlation-Unaware Cache Encoder: Given the descriptions $\left\{W_{0}, W_{1}, W_{2}\right\}$, the cache encoder at the sender computes the Gray-Wyner based cache contents

$$
Z_{r_{i}}=f_{r_{i}}^{\mathfrak{C}_{G W}}\left(\mathrm{~W}_{0}, \mathrm{~W}_{1}, \mathrm{~W}_{2}\right), \quad r_{i} \in\{1,2\} .
$$

- Correlation-Unaware Multicast Encoder: For any demand realization $\mathbf{d}$ revealed to the sender, the GrayWyner based multicast encoder generates and transmits the multicast codeword

$$
Y_{\mathbf{d}}^{G W}=f^{\mathfrak{M}_{G W}}\left(\mathbf{d},\left\{Z_{r_{1}}, Z_{r_{2}}\right\},\left\{\mathrm{W}_{0}, \mathrm{~W}_{1}, \mathrm{~W}_{2}\right\}\right) .
$$

- Multicast Decoder: Receiver $r_{i}$ decodes the descriptions corresponding to its requested file as

$$
\left\{\widehat{\mathrm{W}}_{0}, \widehat{\mathrm{W}}_{d_{r_{i}}}\right\}=g_{r_{i}}^{\mathfrak{M}_{G W}}\left(\mathbf{d}, Y_{\mathbf{d}}^{G W}, Z_{i}\right) .
$$

- Gray-Wyner Decoder: Receiver $r_{i}$ decodes its requested file using the descriptions recovered by the multicast 
decoder, via a mapping $g_{r_{i}}^{G W}:\left[1: 2^{n R_{0}}\right) \times\left[1: 2^{n R_{d_{i}}}\right) \rightarrow$ $\mathcal{X}_{i}^{n}$, as

$$
\widehat{X}_{d_{i}}^{n}=g_{r_{i}}^{G W}\left(\widehat{\mathrm{W}}_{0}, \widehat{\mathrm{W}}_{d_{r_{i}}}\right) .
$$

Notice that for the class of GW-CACM schemes, since $\left(R_{0}, R_{1}, R_{2}\right) \in \mathfrak{S}_{G W}(\mathrm{U})$ and $\left(\mathrm{W}_{0}, \mathrm{~W}_{d_{r_{i}}}\right)$ is a Gray-Wyner description of $X_{d_{r_{i}}}^{n}$, in order to have $\lim _{n \rightarrow \infty} P_{e}^{(n)}=0$, with $P_{e}^{(n)}$ as defined in (1), we only need

$$
\lim _{n \rightarrow \infty} \max _{\mathbf{d}} \max _{r_{i}} \mathbb{P}\left(\left(\widehat{\mathrm{W}}_{0}, \widehat{\mathrm{W}}_{d_{r_{i}}}\right) \neq\left(\mathrm{W}_{0}, \mathrm{~W}_{d_{r_{i}}}\right)\right)=0 .
$$

As in (2), the peak GW-CACM multicast rate is

$$
R_{G W}^{(n)}\left(R_{0}, R_{1}, R_{2}\right)=\max _{\mathbf{d}} \frac{\mathbb{E}\left[L\left(Y_{\mathbf{d}}^{G W}\right)\right]}{n},
$$

where we explicitly show the dependence on $\left(R_{0}, R_{1}, R_{2}\right)$.

In line with Definitions 1 and 2 for a given $U$, the peak U-rate-memory region for the class of GWCACM schemes, $\mathfrak{R}_{G W}^{*}(\mathrm{U})$, is defined as the closure of the union of all the achievable pairs $\left(R_{G W}^{(n)}\left(R_{0}, R_{1}, R_{2}\right), M\right)$ with $\left(R_{0}, R_{1}, R_{2}\right) \in \mathfrak{S}_{G W}(\mathrm{U})$. Analogously, the peak U-ratememory function of GW-CACM, $R_{G W}^{*}(M, \mathrm{U})$, is defined as $R_{G W}^{*}(M, \mathrm{U})=\inf \left\{R:(R, M) \in \mathfrak{R}_{G W}^{*}(\mathrm{U})\right\}$.

We remark that $R_{G W}^{*}(M, \mathrm{U})$ is the rate achieved by a GWCACM scheme with the Gray-Wyner encoder operating at the boundary of the region $\mathfrak{S}_{G W}(\mathrm{U})$. Finally, optimizing over the choice of $\mathrm{U}$, we obtain the peak rate-memory region, $\mathfrak{R}_{G W}^{*}$, and the peak rate-memory function, $R_{G W}^{*}(M)$, as

$$
\mathfrak{R}_{G W}^{*}=c l\left\{\bigcup \mathfrak{R}_{G W}^{*}(\mathrm{U})\right\}, R_{G W}^{*}(M)=\inf R_{G W}^{*}(M, \mathrm{U}),
$$

where $\operatorname{cl}\{\mathcal{S}\}$ denotes the closure of $\mathcal{S}$, and the union and infimum are over all choices of $U$ with $|\mathcal{U}| \leq\left|\mathcal{X}_{1}\right| .\left|\mathcal{X}_{2}\right|+2$.

\section{LOWER BOUNDS}

In this section, we provide lower bounds for $R^{*}(M)$, the optimal peak rate-memory function, and $R_{G W}^{*}(M, \mathrm{U})$, the peak U-rate-memory function of GW-CACM for a given U. The latter bound can also be used to obtain a lower bound for $R_{G W}^{*}(M)$. We then investigate conditions on the cache capacity $M$ under which the lower bounds for $R^{*}(M)$ and $R_{G W}^{*}(M)$ meet. These conditions are then used in Section $\mathrm{V}$ in order to establish the optimality of GW-CACM, and quantify the rate gap from the lower bound as a function of the cache capacity $M$.

\section{A. Lower bound on $R^{*}(M)$}

Theorem 1: For a broadcast caching network with two receivers, cache capacity $M$, and a library composed of two files with joint distribution $p\left(x_{1}, x_{2}\right)$, a lower bound on $R^{*}(M)$, the optimal peak rate-memory function, is given by

$$
\begin{aligned}
R^{L B}(M) & =\inf \{R: \\
R & \geq H\left(\mathrm{X}_{1}, \mathrm{X}_{2}\right)-2 M, \\
R & \geq \frac{1}{2}\left(H\left(\mathrm{X}_{1}, \mathrm{X}_{2}\right)-M\right), \\
R & \left.\geq \frac{1}{2}\left(H\left(\mathrm{X}_{1}, \mathrm{X}_{2}\right)+\max \left\{H\left(\mathrm{X}_{1}\right), H\left(\mathrm{X}_{2}\right)\right\}\right)-M\right\} .
\end{aligned}
$$

Proof: Theorem 1 follows from combining cut-set bounds on $i$ ) the cache-demand-augmented graph, and $i$ ) the timereplication of the cache-demand-augmented graph as described in [7], [12].

Remark 1: The outer bound in Theorem 1 improves the best known bound for correlated sources given in [8. Theorem 2], and when particularized to independent sources, matches the corresponding best known bound derived in [9].

\section{B. Lower bounds on $R_{G W}^{*}(M, \mathrm{U})$ and $R_{G W}^{*}(M)$}

Theorem 2: For a given $\mathrm{U}$, a lower bound on $R_{G W}^{*}(M, \mathrm{U})$, the peak U-rate-memory function of the GW-CACM scheme, is given by

$$
\begin{aligned}
R_{G W}^{L B}(M, \mathrm{U}) & =\inf \{R: \\
R & \geq I\left(\mathrm{X}_{1}, \mathrm{X}_{2} ; \mathrm{U}\right)+H\left(\mathrm{X}_{1} \mid \mathrm{U}\right)+H\left(\mathrm{X}_{2} \mid \mathrm{U}\right)-2 M, \\
R & \geq \frac{1}{2}\left(I\left(\mathrm{X}_{1}, \mathrm{X}_{2} ; \mathrm{U}\right)+H\left(\mathrm{X}_{1} \mid \mathrm{U}\right)+H\left(\mathrm{X}_{2} \mid \mathrm{U}\right)-M\right), \\
R & \geq I\left(\mathrm{X}_{1}, \mathrm{X}_{2} ; \mathrm{U}\right)+H\left(\mathrm{X}_{1} \mid \mathrm{U}\right)+\frac{1}{2} H\left(\mathrm{X}_{2} \mid \mathrm{U}\right)-M, \\
R & \left.\geq I\left(\mathrm{X}_{1}, \mathrm{X}_{2} ; \mathrm{U}\right)+\frac{1}{2} H\left(\mathrm{X}_{1} \mid \mathrm{U}\right)+H\left(\mathrm{X}_{2} \mid \mathrm{U}\right)-M\right\} .
\end{aligned}
$$

Proof: The proof is similar to that of Theorem [1, now applied to Gray-Wyner descriptions at rates $\left(R_{0}, R_{1}, R_{2}\right) \in$ $\mathfrak{S}_{G W}(\mathrm{U})$.

Corollary 1: A lower bound on $R_{G W}^{*}(M)$, the peak ratememory function of GW-CACM, is given by

$$
R_{G W}^{L B}(M)=\inf R_{G W}^{L B}(M, \mathrm{U}),
$$

where the infimum is over all choices of $U$ with $|\mathcal{U}| \leq$ $\left|\mathcal{X}_{1}\right| \cdot\left|\mathcal{X}_{2}\right|+2$.

\section{Where $R_{G W}^{L B}(M)$ and $R^{L B}(M)$ meet}

By comparing the lower bounds in Theorems 1 and 2 , it is easy to see that $R^{L B}(M) \leq R_{G W}^{L B}(M, \mathrm{U})$, and hence, $R^{L B}(M) \leq R_{G W}^{L B}(M)$. In the following, we derive conditions under which $R^{L B}(M)=R_{G W}^{L B}(M)$.

Theorem 3: Let

$$
M_{1} \triangleq \max _{X_{1}-U-X_{2}} \frac{1}{2} \min \left\{H\left(\mathrm{X}_{1} \mid \mathrm{U}\right), H\left(\mathrm{X}_{2} \mid \mathrm{U}\right)\right\} .
$$

Then, for $M \in\left[0, M_{1}\right] \cup\left[H\left(\mathrm{X}_{1}, \mathrm{X}_{2}\right)-2 M_{1}, H\left(\mathrm{X}_{1}, \mathrm{X}_{2}\right)\right]$, we have $R_{G W}^{L B}(M)=R^{L B}(M)$.

Proof: Theorem 3 follows from comparing $R^{L B}(M)$ with $R_{G W}^{L B}(M, \mathrm{U})$ for a given $\mathrm{U}$, over different regions of memory $M$. It is observed that when

$$
\begin{aligned}
& M \in\left[0, \frac{1}{2} \min \left\{H\left(\mathrm{X}_{1} \mid \mathrm{U}\right), H\left(\mathrm{X}_{2} \mid \mathrm{U}\right)\right\}\right] \bigcup \\
& \quad\left[H\left(\mathrm{X}_{1}, \mathrm{X}_{2}\right)-\min \left\{H\left(\mathrm{X}_{1} \mid \mathrm{U}\right), H\left(\mathrm{X}_{2} \mid \mathrm{U}\right)\right\}, H\left(\mathrm{X}_{1}, \mathrm{X}_{2}\right)\right],
\end{aligned}
$$

$R_{G W}^{L B}(M, \mathrm{U})-R^{L B}(M)$ is independent from the cache capacity $M$, and becomes zero when $I\left(\mathrm{X}_{1}, \mathrm{X}_{2} ; \mathrm{U}\right)+H\left(\mathrm{X}_{1} \mid \mathrm{U}\right)+$ $H\left(\mathrm{X}_{2} \mid \mathrm{U}\right)=H\left(X_{1}, X_{2}\right)$. For the choice of $\mathrm{U}$ used to obtain $M_{1}$, the region of memory over which the two bounds meet is maximized. 
Remark 2: The Markov chain $X_{1}-U-X_{2}$ in Theorem 3 suggests that for the rate triplet $\left(R_{0}, R_{1}, R_{2}\right) \in \mathfrak{S}_{G W}(\mathrm{U})$ used in GW-CACM, we require $R_{0}+R_{1}+R_{2}=H\left(X_{1}, X_{2}\right)$. The same Markov chain is also used to define Wyner's common information [11]. While in Wyner's common information the goal is to minimize $R_{0}$ subject to $R_{0}+R_{1}+R_{2}=H\left(X_{1}, X_{2}\right)$, for $M_{1}$ in Theorem 3 , the goal is to maximize $\min \left(R_{1}, R_{2}\right)$.

Corollary 2: If the file library $\left(X_{1}, X_{2}\right)$ is such that

$$
\left(I\left(\mathrm{X}_{1} ; \mathrm{X}_{2}\right), H\left(\mathrm{X}_{1} \mid \mathrm{X}_{2}\right), H\left(\mathrm{X}_{2} \mid \mathrm{X}_{1}\right)\right) \in c l\left\{\bigcup \mathfrak{S}_{G W}(\mathrm{U})\right\},
$$

where the union is over all choices of $U$ with $|\mathcal{U}| \leq\left|\mathcal{X}_{1}\right| \cdot\left|\mathcal{X}_{2}\right|+$ 2 , then $R^{L B}(M)=R_{G W}^{L B}(M)$.

Proof: See [13].

Example 1: Consider a 2-DMS whose joint pmf $p\left(x_{1}, x_{2}\right)$ is such that $\left(\mathrm{X}_{1}, \mathrm{X}_{2}\right)$ can be represented as $\mathrm{X}_{1}=\left(\mathrm{X}_{1}^{\prime}, \mathrm{V}\right)$ and $\mathrm{X}_{2}=\left(\mathrm{X}_{2}^{\prime}, \mathrm{V}\right)$, where $\mathrm{X}_{1}^{\prime}$ and $\mathrm{X}_{2}^{\prime}$ are conditionally independent given $\mathrm{V}$. Taking $\mathrm{U}=\mathrm{V}$, the point $R_{0}=I\left(\mathrm{X}_{1}, \mathrm{X}_{2} ; \mathrm{U}\right)=$ $H(\mathrm{~V})=I\left(\mathrm{X}_{1} ; \mathrm{X}_{2}\right), R_{1}=H\left(\mathrm{X}_{1} \mid \mathrm{U}\right)=H\left(\mathrm{X}_{1}^{\prime} \mid \mathrm{V}\right)=H\left(\mathrm{X}_{1} \mid \mathrm{X}_{2}\right)$ and $R_{2}=H\left(\mathrm{X}_{2} \mid \mathrm{U}\right)=H\left(\mathrm{X}_{2}^{\prime} \mid \mathrm{V}\right)=H\left(\mathrm{X}_{2} \mid \mathrm{X}_{1}\right)$ belongs to the Gray-Wyner rate region. Then, by Theorem 2. $R^{L B}(M)=$ $R_{G W}^{L B}(M)$ for any $M$.

\section{AN ACHIEVABLE SCHEME BASED ON GW-CACM AND ITS OPTIMALITY}

In this section, we present an achievable GW-CACM scheme, where $i$ ) the first step consists of a Gray-Wyner encoder restricted to operate on the plane of the Gray-Wyner rate region with $R_{1}=R_{2}=\rho$, and $i i$ ) the second step is a deterministic correlation-unaware multiple-request CACM scheme that combines ideas from conventional caching (e.g., $\mathrm{LFU}^{2}$ and uncoded multicasting) and correlation-unaware CACM with coded placement, as suggested by Tian and Chen in [14], and referred to as TC in the following. We remark that jointly optimizing these two steps is the key to maximizing the overall performance.

We then refer to the overall scheme as GW-LFU-TC, which works as follows:

Gray-Wyner Encoder: generates three library descriptions $\left\{\mathrm{W}_{0}, \mathrm{~W}_{1}, \mathrm{~W}_{2}\right\}$ using a conditional pmf $p\left(u \mid x_{1}, x_{2}\right)$ such that $p\left(x_{1} \mid u\right)=p\left(x_{2} \mid u\right)$ with $\left(R_{0}, \rho, \rho\right) \in \mathfrak{S}_{G W}(\mathrm{U})$.

Cache Encoder: populates the receiver caches as:

- If $M \in[0, \rho)$, the common description $\mathrm{W}_{0}$ is not cached at either receiver, and descriptions $\left\{W_{1}, W_{2}\right\}$ are cached according to the caching phase of TC.

- If $M \in\left[\rho, R_{0}+\rho\right)$, the first $n(M-\rho)$ bits of description $\mathrm{W}_{0}$ are cached at both receivers (as per LFU caching), and descriptions $\left\{W_{1}, W_{2}\right\}$ are cached according to TC over the remaining cache capacity $\rho$.

- If $M \in\left[R_{0}+\rho, R_{0}+2 \rho\right]$, the common description $\mathrm{W}_{0}$ is fully cached at both receivers, and descriptions $\left\{W_{1}, W_{2}\right\}$ are cached according to TC over the remaining cache capacity $M-R_{0}$.

${ }^{2} \mathrm{LFU}$ is a local caching policy that, in the setting of this paper, leads to all receivers caching the same part of the file.
Multicast Encoder: transmits the descriptions $\left\{W_{1}, W_{2}\right\}$ according to conventional coded multicast schemes [1]-[3], [14], while the portion of $W_{0}$ missing at each receiver cache is transmitted via uncoded (naive) multicast.

Remark 3: Differently from the single-cache setting analyzed in [4], where caching the common description first is always optimal, in our case, when the cache capacity is smaller than the private description size, $\rho$, it is optimal to first cache the private descriptions.

Theorem 4: Given a conditional pmf $p\left(u \mid x_{1}, x_{2}\right)$ such that $p\left(x_{1} \mid u\right)=p\left(x_{2} \mid u\right)$, a cache capacity $M$, and a rate triplet $\left(R_{0}, \rho, \rho\right) \in \mathfrak{S}_{G W}(\mathrm{U})$, the peak U-rate achieved by GW-LFU$\mathrm{TC}$ is given by

$$
R_{G W}^{U B}(M, \mathrm{U})=\inf R_{a c h}\left(R_{0}, \rho\right),
$$

where the infimum is over all rate triplets $\left(R_{0}, \rho, \rho\right) \in$ $\mathfrak{S}_{G W}(\mathrm{U})$, and $R_{a c h}\left(R_{0}, \rho\right)$ is

$R_{a c h}\left(R_{0}, \rho\right)= \begin{cases}R_{0}+2 \rho-2 M, & M \in\left[0, \frac{1}{2} \rho\right) \\ R_{0}+\frac{3}{2} \rho-M, & M \in\left[\frac{1}{2} \rho, R_{0}+\rho\right) \\ \frac{1}{2} R_{0}+\rho-\frac{1}{2} M, & M \in\left[R_{0}+\rho, R_{0}+2 \rho\right] .\end{cases}$

Furthermore, optimizing over $U$, the peak rate achieved by GW-LFU-TC is given by

$$
R_{G W}^{U B}(M)=\inf R_{G W}^{U B}(M, \cup),
$$

where the infimum is over all choices of $U$ with $|\mathcal{U}| \leq$ $\left|\mathcal{X}_{1}\right| .\left|\mathcal{X}_{2}\right|+2$.

Proof: See [13].

\section{A. Optimality of $G W-L F U-T C$}

In order to prove the optimality of the GW-LFU-TC scheme, we first state the following theorem:

Theorem 5: For any $\mathrm{U}$ and $M$,

$$
R_{G W}^{U B}(M, \mathrm{U})=R_{G W}^{*}(M, \mathrm{U}) .
$$

Proof: Similar to the proof of Theorem 3, $R_{G W}^{U B}(M, \mathrm{U})$ is compared to the lower bound $R_{G W}^{L B}(M, \mathrm{U})$ in each memory region, and for any $\mathrm{U}$ forming a Markov chain, $\mathrm{X}_{1}-\mathrm{U}-\mathrm{X}_{2}$.

Example 2: Assuming the same setting as in Example 1 . since $R^{L B}(M)=R_{G W}^{L B}(M)$ for any $M$, it follows from Theorem 5 that the GW-LFU-TC scheme is optimal for all values of memory $M$.

The following theorem characterizes the performance of the GW-LFU-TC scheme for different regions of $M$, and delineates the cache capacity region for which the scheme is optimal or near optimal.

Theorem 6: Let

$$
\widetilde{M}_{1} \triangleq \max _{X_{1}-U-X_{2}} \frac{1}{2} \min \left\{H\left(\mathrm{X}_{1} \mid \mathrm{U}\right), H\left(\mathrm{X}_{2} \mid \mathrm{U}\right)\right\},
$$

where the max is over all choices of $U$ such that $p\left(x_{1} \mid u\right)=$ $p\left(x_{2} \mid u\right)$.

Then, for $M \in\left[0, \widetilde{M}_{1}\right] \cup\left[H\left(\mathrm{X}_{1}, \mathrm{X}_{2}\right)-2 \widetilde{M}_{1}, H\left(\mathrm{X}_{1}, \mathrm{X}_{2}\right)\right]$, the GW-LFU-TC scheme is optimal i.e., $R_{G W}^{U B}(M)=R^{*}(M)$. 
In addition, for $M \in\left(\widetilde{M}_{1}, H\left(\mathrm{X}_{1}, \mathrm{X}_{2}\right)-2 \widetilde{M}_{1}\right)$, we have

$$
R_{G W}^{U B}(M)-R^{*}(M) \leq \frac{1}{2} \min \left\{H\left(\mathrm{X}_{1} \mid \mathrm{X}_{2}\right), H\left(\mathrm{X}_{2} \mid \mathrm{X}_{1}\right)\right\}-\widetilde{M}_{1} \text {. }
$$

Proof: See [13]

\section{B. Illustration of Results: Doubly Symmetric Binary Source}

Consider, as a 2-DMS, a doubly symmetric binary source (DSBS) with joint pmf $p\left(x_{1}, x_{2}\right)=\frac{1}{2}\left(1-p_{0}\right) \delta_{x_{1}, x_{2}}+\frac{1}{2} p_{0}(1-$ $\left.\delta_{x_{1}, x_{2}}\right), x_{1}, x_{2} \in\{0,1\}$, and parameter $p_{0} \in\left[0, \frac{1}{2}\right]$. Then,

$$
\begin{aligned}
& H\left(\mathrm{X}_{1}\right)=H\left(\mathrm{X}_{2}\right)=1, \\
& H\left(\mathrm{X}_{1} \mid \mathrm{X}_{2}\right)=H\left(\mathrm{X}_{2} \mid \mathrm{X}_{1}\right)=h\left(p_{0}\right), \\
& H\left(\mathrm{X}_{1}, \mathrm{X}_{2}\right)=1+h\left(p_{0}\right),
\end{aligned}
$$

where $h(p)=-p \log (p)-(1-p) \log (1-p)$ is the binary entropy function. As derived in [11], an achievable Gray-Wyner rate region of a DSBS restricted to the plane $\left\{\left(R_{0}, R_{1}, R_{2}\right): R_{1}=R_{2}=\rho\right\}$, is described by the set of rate triplets $\left(R_{0}, \rho, \rho\right)$ with $R_{0}$ given by

$$
R_{0} \geq \begin{cases}1+h\left(p_{0}\right)-2 \rho, & 0 \leq \rho<h\left(p_{1}\right) \\ f(\rho) & h\left(p_{1}\right) \leq \rho \leq 1\end{cases}
$$

where $p_{1}=\frac{1}{2}\left(1-\sqrt{\left(1-2 p_{0}\right)}\right)$,

$$
\begin{aligned}
& f(\rho) \triangleq 1+h\left(p_{0}\right)+\left(h^{-1}(\rho)-\frac{p_{0}}{2}\right) \log \left(h^{-1}(\rho)-\frac{p_{0}}{2}\right)+ \\
& p_{0} \log \left(\frac{p_{0}}{2}\right)+\left(1-h^{-1}(\rho)-\frac{p_{0}}{2}\right) \log \left(1-h^{-1}(\rho)-\frac{p_{0}}{2}\right),
\end{aligned}
$$

and $h^{-1}(\rho)$ is the inverse of the binary entropy function.

We compare the performance of the proposed GW-LFU-TC scheme with respect to: 1) LFU caching with uncoded multicasting (LFU-UM), 2) the deterministic correlation-unaware CACM in [14], referred to as TC, 3) the lower bound on the GW-CACM peak rate-memory function $\left(R_{G W}^{L B}\right)$, and 4) the lower bound on the optimal peak rate-memory function $\left(R^{L B}\right)$. Fig. 2 displays the rate-memory trade-offs for $p_{0}=0.2$.

In line with Theorems 3 and 6, Fig. 2 shows that the lower bound on the Gray-Wyner rate-memory function $\left(R_{G W}^{L B}\right)$ coincides with the lower bound on the optimal rate-memory function $\left(R^{L B}\right)$ for $M \leq \widetilde{M}_{1}=0.25$ and $M \geq\left(H\left(\mathrm{X}_{1}, \mathrm{X}_{2}\right)-\right.$ $\left.2 \widetilde{M}_{1}\right)=1.22$, and GW-LFU-TC is optimal in this region, while correlation-unaware schemes, LFU-UM and TC, fall short. Furthermore, the gap between the rate achieved with GW-LFU-TC and the optimal peak rate-memory function is less than 0.11 , which is less than half of the conditional entropy, 0.36. Finally, in line with Theorem 5. GW-LFU-TC achieves $R_{G W}^{L B}$ for any $M$.

\section{CONCLUding REMARKS}

In this paper, we have studied the fundamental limits of cache-aided communication systems under the assumption of correlated content for a two-user two-file network. We have derived a lower bound on the peak rate-memory function for such systems and proposed a class of schemes based on a twostep source coding approach. Files are first compressed using Gray-Wyner source coding, and then cached and delivered

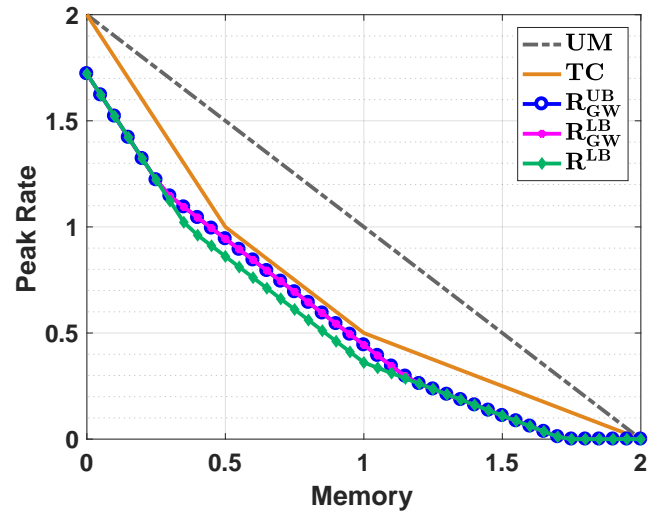

Fig. 2: Rate-memory trade-off for a DSBS with $p_{0}=0.2$.

using a combination of existing correlation-unaware cached aided coded multicast schemes. We have fully characterized the rate-memory trade-off of such class of schemes, proposed an achievable two-step scheme, and proved its optimality for different memory regimes. Finally, in [13], we provide an extended analysis that includes the characterization of both peak and average rate-memory trade-offs in more general userfile settings.

\section{ACKNOWLEDGEMENT}

The authors would like to thank M. Wigger and D. Gündüz for their useful discussions on the Gray-Wyner network.

\section{REFERENCES}

[1] M. A. Maddah-Ali and U. Niesen, "Fundamental limits of caching," IEEE Transactions on Information Theory, vol. 60, no. 5.

[2] M. Ji, A. Tulino, J. Llorca, and G. Caire, "Order-optimal rate of caching and coded multicasting with random demands," arXiv:1502.03124, 2015.

[3] — "Caching-aided coded multicasting with multiple random requests," in Proc. IEEE Information Theory Workshop (ITW), 2015.

[4] R. Timo, S. S. Bidokhti, M. Wigger, and B. C. Geiger, "A rate-distortion approach to caching," arXiv preprint arXiv:1610.07304, 2016.

[5] P. Hassanzadeh, A. Tulino, J. Llorca, and E. Erkip, "Cache-aided coded multicast for correlated sources," Proc. IEEE International Symposium on Turbo Codes and Iterative Information Processing (ISTC), 2016.

[6] _ , "Correlation-aware distributed caching and coded delivery," Proc. IEEE Information Theory Workshop (ITW), 2016.

[7] J. Llorca, A. M. Tulino, K. Guan, and D. Kilper, "Network-coded caching-aided multicast for efficient content delivery," in Proc. IEEE International Conference on Communications (ICC).

[8] S. H. Lim, C.-Y. Wang, and M. Gastpar, "Information theoretic caching: The multi-user case," in Proc. IEEE International Symposium on Information Theory (ISIT), 2016, pp. 525-529.

[9] H. Ghasemi and A. Ramamoorthy, "Improved lower bounds for coded caching," in Proc. IEEE International Symposium on Information Theory (ISIT), 2015, pp. 1696-1700.

[10] R. Gray and A. Wyner, "Source coding for a simple network," Bell System Technical Journal, 1974.

[11] A. Wyner, "The common information of two dependent random variables," IEEE Transactions on Information Theory, 1975.

[12] J. Llorca and A. M. Tulino, "Minimum cost caching-aided multicast under arbitrary demand," in Proc. IEEE Asilomar Conference on Signals, Systems and Computers, 2013, pp. 236-237.

[13] P. Hassanzadeh, A. Tulino, J. Llorca, and E. Erkip, "Rate-memory tradeoff for the broadcast caching network with correlated sources," preprint at http://engineering.nyu.edu/eelab/people/Parisa.

[14] C. Tian and J. Chen, "Caching and delivery via interference elimination," in Proc. IEEE International Symposium on Information Theory (ISIT), 2016. 